\title{
Reconstruction of Complex Abdominal Wall Defects
}

\author{
Brig AS Bath, SM, vsm*, Col PK Patnaik ${ }^{+}$, Lt Col PS Bhandari"
}

\begin{abstract}
Introduction:Reconstruction of large abdominal wall defects not amenable to primary closure remains a challenging problem. These defects result from trauma, previous surgery, infection and tumour resection. The primary objectives of abdominal wall reconstructions are to protect abdominal contents and provide functional support. The abdominal wall reconstruction aims at providing basic component parts, i.e. skin, soft tissue and fascia. For large soft tissue defects, pedicled or free flap closure can be used. In clean wounds, fascial replacement is accomplished with synthetic mesh provided there is adequate soft tissue coverage. Methods: We treated a total of 20 consecutive patients with complex abdominal wall defects utilizing various reconstructive procedures. There were 15 males $(75 \%)$ and 5 females $(25 \%)$. The aetiology included dehiscence of laparotomy wounds in eight $(40 \%)$, following ablative surgery for malignant tumours in seven (35\%), trauma in three (15\%) and congenital defects in two $(\mathbf{1 0} \%)$ cases. The reconstructive procedures consisted of onlay prolene mesh in seven (35\%), Gore-Tex (PTFE) dual mesh both as inlay and onlay in five $(\mathbf{2 5 \%})$, facial partition release technique in three $(\mathbf{1 5 \%})$, inlay prolene mesh covered with omentum and split skin graft in two (10\%), inlay prolene mesh covered with expanded skin in two (10\%), and Gore-Tex dual mesh covered with latissimus dorsi myocutaneous flap in one $(5 \%)$ case. Postoperatively none developed mesh infection or extrusion. Three patients with malignant aetiology received postoperative radiotherapy. During follow up, one patient developed ventral hernia cephalad to the repair and one died due to recurrence of abdominal wall malignancy.

Conclusion: The reconstruction of an abdominal wall defect requires a comprehensive plan of preoperative and post operative care of the patient and aims toward restoration of abdominal structural integrity by a variety of procedures. The use of new biomaterials and tissue expanders provides reliable and durable abdominal wall closure along with good aesthetic results.
\end{abstract}

MJAFI 2007; 63 : 123-126

Key Words: Abdominal wall defect; Mesh repair; Abdominal wall reconstruction

\section{Introduction}

$\mathrm{A}$ complex abdominal defect is considered to be one, which defies primary anatomical repair without tension. This defect may be due to gross tissue loss, multiple previous procedures or distorted anatomy. Numerous methods are available for reconstruction of complex abdominal wall defects depending upon the clinical situation, which include presence or absence of infection, presence of ostomies, location and size of defect. The aims of abdominal wall reconstruction are protection and cover of abdominal viscera, repair and prevention of abdominal wall herniation and to achieve acceptable surface contour.

\section{Material and Methods}

During last six years (May 1997 to April 2003), twenty patients with complex abdominal wall defects were reconstructed with a variety of procedures. The age group ranged from 10 to 70 years (Table 1) and most cases occurred after dehiscence of laparotomy wound (Table 2). The congenital abdominal defects were in the form of omphalocele. In $80 \%$, the defects were located in the anterior abdominal wall and involved more than two quadrants of abdomen.
The selection of operative procedure depended upon the clinical situation, size and location of defect, presence of ostomies and availability of skin cover (Table 3). Fascial partition release technique was used for repair of ventral wall hernia where defect in linea alba in umbilical region was less than $12 \mathrm{~cm}$. Bilateral parallel pararectal incision into external oblique aponeurosis were given and rectus muscle along with anterior rectus sheath was moved medially and repair was performed with loop nylon sutures. The repair was further supported by nylon darn of anterior rectus sheath.

Five patients in ventral wall hernia group had abdominal wall dehiscence in the immediate post-operative period. They had wound infection, visceral oedema and their general condition was poor. In these patients staged abdominal wall reconstruction was done. In the first stage, as an emergency

Table 1

Age group

Age Number

10-20 years 2

$20-40$ years 11

40-50 years 03

50-70 years 04

\footnotetext{
*Commandant, Military Hospital (CTC) Pune-411040. ${ }^{+}$Senior Advisor (Surgery \& GI Surgery), Command Hospital (WC) Chandimandir. ${ }^{\#}$ Classified Specialist (Surgery \& Reconstructive Surgery), Army Hospital (R\&R), Delhi Cantt-110010.
} 
Table 2

Aetiology of Abdominal Wall Defect

Aetiology

Number

Congenital

02

Trauma

03

Tumor resection

Ventral wall hernias

measure split skin was applied over exposed viscera and once skin graft had taken up, the patient was given abdominal belt to control ventral hernia. Two of these patients also had colostomy opening over the abdominal wall (Fig.1). Second stage was performed 6 to 10 months later, when reconstruction of abdominal wall defect was performed after excision of the skin graft. Colostomy closure was also done at the same sitting(Fig.2). Onlay Gore-Tex (PTFE) dual mesh was used in two patients and in remaining three onlay Prolene (Polypropylene) mesh was used for repair of musculofascial defect. The mesh was fixed with remaining musculofascial layer using multiple rows of prolene sutures. In cases of post traumatic abdominal wall defects the initial repair was done with split skin grafts. Subsequently tissue expansion over a period of 8-10 weeks was performed and once adequate skin expansion was achieved, the skin graft was excised and the expanded skin was used for obtaining skin closure over the mesh.

In the tumour resection group, inlay prolene mesh was used directly over abdominal viscera in three patients. Prolene mesh was further covered with island omental flap in two patients, over which split skin graft was applied. In the remaining four patients inlay Gore-Tex dual mesh was used for repair of musculofascial defect and one of these patients required latissimus dorsi myocutaneous flap to cover the mesh. In the congenital group onlay prolene mesh was used for repair of musculofascial defect and lower abdominal skin was mobilized to cover the mesh.

\section{Result}

Twenty patients underwent reconstruction of complex abdominal wall defects. Dehiscence of laparotomy wound was the cause of abdominal defect in majority $(40 \%)$ of the cases. A variety of procedures were used to reconstruct the defects. Most of the patients (35\%) required reconstruction with onlay prolene mesh.

Complications occurred in three patients.One patient developed postoperative wound infection. She was an obese patient who had fascial partition release procedure for recurrent ventral hernia. During surgery she was found to be having small abscess around previous sutures. The infection settled with culture specific antibiotics and she did not develop recurrent ventral hernia. Another patient had superficial necrosis of skin flap without exposure of mesh and after three years she developed another ventral hernia cephalad to previous mesh repair. One patient with abdominal wall tumor developed recurrence of malignancy and died after seven months. All other patients had uneventful recovery without recurrence of hernia (Fig. 3).

Table 3

Procedures for Reconstruction

Procedure Number

Fascial partition release 03

Onlay prolene mesh 07

Prolene mesh + omentum + S.S.G. 02

Prolene mesh + tissue expansion $\quad 02$

Onlay / inlay gore-tex mesh 05

Gore-tex mesh +myocutaneous flap $\quad 01$

\section{Discussion}

The common clinical problems requiring abdominal wall reconstruction include congenital defects like omphalocele, necrotising fascitis, tumour resection, trauma and ventral wall hernias following dehiscence of laparotomy wound. In our series ventral wall hernias and full thickness abdominal wall defects following resection of tumours accounted for $75 \%$ of complex abdominal wall defects. Anatomical repair consisting of re-approximation of linea alba without tension is the ideal goal [1], however this is difficult in cases of complex abdominal wall defects due to multiple previous procedures, distorted anatomy and gross tissue loss.

In acute setting of an abdominal wall dehiscence, a split skin graft can be applied over exposed viscera or over an absorbable mesh. In this series there were five cases from this group, however no mesh was used under the skin graft. During second surgery for repair of the large ventral hernia, hardly any adhesions were found between skin graft and viscera and synthetic mesh was used as an onlay graft for repair of abdominal wall defect. Some authors [2,3] have described advantages of retrorectus placement of mesh as an inlay graft but we found that retrorectus dissection to be traumatic particularly in cases with multiple previous abdominal operations, besides our results of onlay placement of mesh have been comparable to inlay placement of mesh.

The technique of facial partition release can be used for closure of large abdominal wall defects(up to $20 \mathrm{~cm}$ wide) in the umbilical region [4], however we were unable to close large defects with this technique and limited the use of this procedure for defects of up to 12 $\mathrm{cm}$ width. The advantage of this technique is minimal use of foreign material and there is no risk of extrusion of synthetic mesh even if postoperative infection occurs.

Biomaterials as an inlay mesh, have a major role to play in full thickness abdominal wall defects following tumour resection. Polytetrafluoroethylene (Gore-Tex) has been reported [5], to be the ideal material for this purpose as no complications relating to adhesions or erosion into viscera have been reported with this material and it has low foreign body reaction. However prior to availability of this material, we have used inlay 


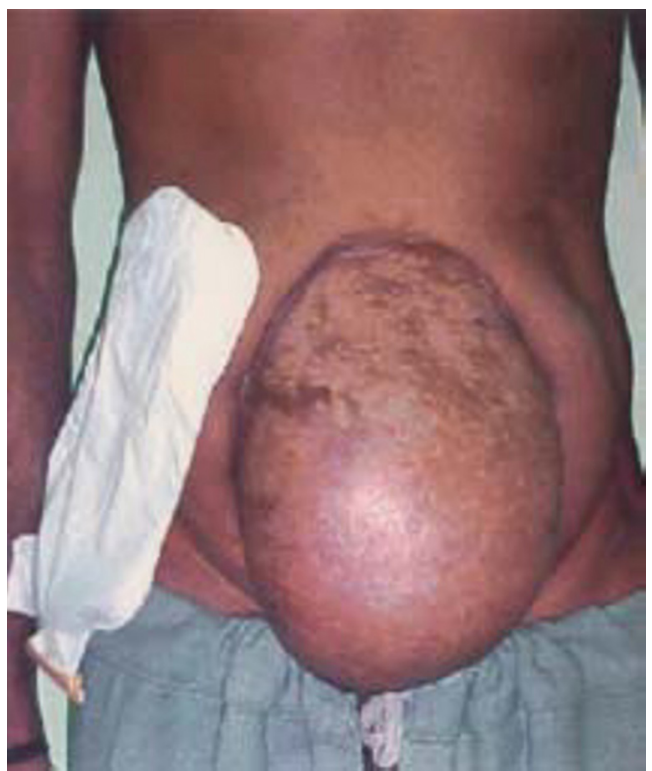

Fig. 1 : Preoperative photograph showing massive ventral hernia with colostomy

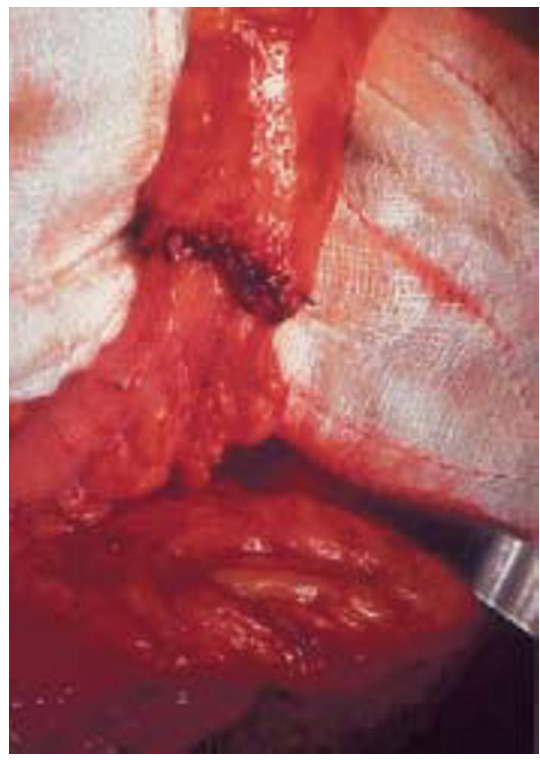

Fig. 2: Operative photograph showing completed colostomy closure

polypropylene (Prolene) mesh without any problem of bowel fistula and we feel that this material can still be used directly over abdominal viscera if no adhesion lysis has been performed. The advantages of prolene mesh are porosity and good adhesions to musculofascial layer resulting in lower chances of recurrent herniation particularly when it is used as an onlay mesh and it is cheaper than Gore-Tex mesh [6]. We have restricted the use of Gore-Tex mesh to cases where alloplastic material is to be used directly over abdominal viscera, post-operative radiotherapy will be required and in cases where contamination of operative field is likely to occur e.g concomitant colostomy closure. The disadvantages of Gore-Tex mesh are poor fixation to musculofascial layer due to poor tissue ingrowth resulting in higher

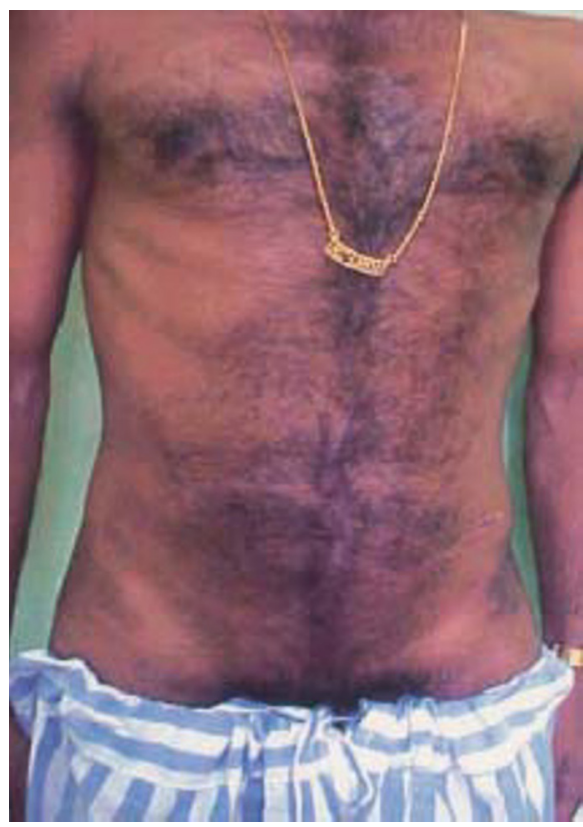

Fig. 3 : Postoperative photograph at two years

incidence of reherniation and high cost of this material. The use of synthetic mesh requires adequate soft tissue coverage to avoid complications [7]. We have resorted to use of island omental flap or expanded skin whenever native skin and soft tissues are inadequate to cover the alloplastic material. Tissue expansion gives good results in terms of colour match, contours and donor site morbidity $[8,9]$. The disadvantage is that it requires multiple stages and uncontaminated operative field.

Various muscle and myocutaneous flaps like tensor fascia lata [10], rectus femoris [11], rectus abdominis [12] and latissimus dorsi [13] have been reported in literature for repair of abdominal wall defects. In this series we have used pedicled latissimus dorsi myocutaneous flap in one case only to cover Gore-Tex mesh for the patient requiring postoperative radiotherapy.

With the availability of new biomaterials like polytetrafluoroethylene (Gore-Tex) and tissue expanders [14], the requirement of complex flaps along and the donor site morbidity can be significantly reduced.

\section{Conflicts of Interest}

None identified

\section{References}

1. Cassar K, Munro A. Surgical treatment of incisional hernia. Br J Surg 2002; 89: 534-45.

2. McLanahan D, King LT, Weems C, et al. Retrorectus prosthetic mesh repair of a midline abdominal hernia. Am J Surg 1997; 173: 445.

3. Moscona RA, Ramon Y, Toledano H, et al. Use of synthetic mesh for the interabdominal wall after TRAM flap transfer. Plast Reconstr Surg 1998;101:706-10.

4. Kara IG, Erdenm E, Nessar M, et al. Reconstruction of large 
abdominal wall defects. Eur J Plast Surg 1999;22: 394-8.

5. Chrysos E, Athanasakis E, Kafetzakis A, Dimitriadou D, Koutsoumpas V, et al. Surgical repair of incision hernias: tension free technique using prosthetic materials (expanded polytetrafluroethylene Gore-Tex Dual mesh). Ann Surg 2000; 66: 679- 82 .

6. Ladurner R, Trupka A, Schmidbauer S, Hallfeldt K. The use of an underlay polypropylene mesh in complicated incisional hernias: successful French surgical technique. Minerva chir 2001; 56: 111-7.

7. Mathes SJ, Steinwald PM, Foster RD, et al. Complex abdominal wall reconstruction: A comparison of flap and mesh closure. Ann Surg 2000;232:586-90.

8. Jacobsen WM, Petty PM, Bite U, et al. Massive abdominal wall hernia reconstruction with expanded external/internal oblique and transversalis musculofascia. Plast Reconstr Surg 1997; 100: 326.

9. Okunski WJ, Sonntag BV, Murphy RX. Staged reconstruction of abdominal wall defects after intra abdominal catastrophes. Ann Plast Surg 1996; 36: 475.

10. Williams JK, Carlson GW, deChalain T, et al. The role of tensor fascia lata in abdominal wall reconstruction. Plast Reconstr Surg 1998; 101: 713.

11. Caulfield WH, Curtsinger I, Powell G, et al. Donor leg morbidity after pedicled rectus femoris muscle flap transfer for abdominal wall and pelvic reconstruction. Ann Plast Surg 1994;32:37782 .

12. DeFranzo AJ, Kingman GJ, Sterchi JM, et al. Rectus turnover flaps for the reconstruction of large abdominal wall defects. Ann Plast Surg 1996; 37:18-23.

13. Ninkovic M, Kronberger P, Harpf C, et al. Free innervated latissimus dorsi musculocutaneous flap for reconstruction of full thickness abdominal wall defects. Plast Reconstr Surg 1998;101:971-5.

14. Carlson G W, Elwood E, Losken A, Galloway JR. The role of tissue expansion in abdominal wall reconstruction. Ann Plast Surg 2000; 44: 147-53.

\section{CORPS NEWS}

The following award winning papers were presented during the 55th AFMRC, held on 6th Feb 2007 at Armed Forces Medical College, Pune:

\section{Chief of Army Staff Award-2006}

Title: Analysis of HIV Type -1 subtype full length GAG gene sequence from India - Novel observations and plausible implications

Speaker: Lt Col RM Gupta, Reader, Dept of Microbiology, Armed Forces Medical College, Pune.

2. Chief of Naval Staff Award-2006

Title: Impact of Hepatitis C Virus Infection in Renal Transplant Recipients

Speaker: Col AS Narula, Professor and Head, Department of Internal Medicine, Armed Forces Medical College, Pune.

\section{Chief of Air Staff Award-2006}

Title: Management of Rh Isoimmunised Pregnancies

Speaker: Lt Col Devendra Arora, Reader, Department of Obstetric and Gynaecology, Armed Forces Medical College, Pune.

4. DGAFMS \& Senior Colonel Commandant Award-2006

Title: Aircrew Ejection Experience: Questionnaire Responses from 20 Survivors

Speaker: Wg Cdr N Taneja, Classified Specialist (Aviation Medicine), Institute of Aerospace Medicine, Bangalore

5. Late Lt Gen RS Hoon, PVSM, AVSM Award-2006

Title: Transfer for Primary Angioplasty in Acute Myocardial Infarction-Safe and Effective

Speaker: Wg Cdr H Madan, Classified Specialist (Medicine and Cardiology), Command Hospital (AF), Bangalore 